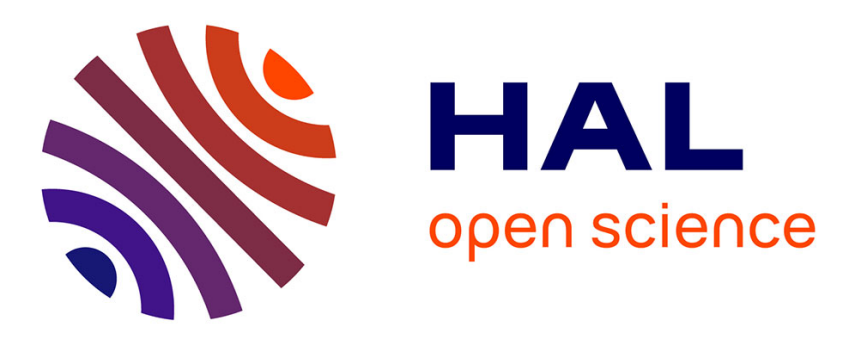

\title{
Uncertainty quantification in computational nonlinear elasticity
}

Evangéline Capiez-Lernout, Christian Soize, M. P. Mignolet

\section{To cite this version:}

Evangéline Capiez-Lernout, Christian Soize, M. P. Mignolet. Uncertainty quantification in computational nonlinear elasticity. ASME 2012 11th Biennal Conference on Engineering Systems Design and Analysis, Jul 2012, Nantes, France. Paper ESDA2012-82246, Pages : 1-10. hal-00734157

\section{HAL Id: hal-00734157 \\ https://hal.science/hal-00734157}

Submitted on 20 Sep 2012

HAL is a multi-disciplinary open access archive for the deposit and dissemination of scientific research documents, whether they are published or not. The documents may come from teaching and research institutions in France or abroad, or from public or private research centers.
L'archive ouverte pluridisciplinaire HAL, est destinée au dépôt et à la diffusion de documents scientifiques de niveau recherche, publiés ou non, émanant des établissements d'enseignement et de recherche français ou étrangers, des laboratoires publics ou privés. 
Proceedings of ASME 2012 11th biennal conference on engineering systems design and
analysis
ESDA 2012

\section{ESDA2012-82246}

\section{UNCERTAINTY QUANTIFICATION IN COMPUTATIONAL NONLINEAR ELASTICITY.}

\author{
E. Capiez-Lernout* \\ Université Paris-Est \\ Modélisation et Simulation \\ Multi Echelle \\ MSME UMR 8208 CNRS \\ 5 bd Descartes \\ F-77455 Marne-la-Vallée, France \\ Email:
}

\author{
C. Soize \\ Université Paris-Est \\ Modélisation et Simulation \\ Multi Echelle \\ MSME UMR 8208 CNRS \\ 5 bd Descartes \\ F-77455 Marne-la-Vallée, France \\ Email: christian.soize@univ-paris-est.fr
}

\author{
M.-P. Mignolet \\ SEMTE \\ Faculties of Mechanical \\ and Aerospace Engineering \\ Arizona State University, Tempe \\ Arizona 85287-6106, USA \\ Email : marc.mignolet@asu.edu
}

evangeline.capiezlernout@univ-paris-est.fr

\begin{abstract}
This work presents a methodology for the construction of an uncertain nonlinear computational model adapted to the static analysis of a complex mechanical system. The deterministic nonlinear computational model is constructed with the finite element method using a total Lagrangian formulation. The finite element nonlinear response is then considered as a reference deterministic solution from which a reduced-order basis is constructed using the POD (Proper Orthogonal Decomposition) methodology. The mean reduced nonlinear computational model is thus obtained by projecting the reference deterministic solution on this basis. The explicit construction of the mean reduced nonlinear computational model is proposed for any type of structure modeled with three-dimensional solid finite elements. A procedure for the robust identification of the uncertain nonlinear computational model with respect to experimental responses is then given. Finally, the methodology is applied to a structure for which simulated experiments are given.
\end{abstract}

\section{INTRODUCTION}

In structural mechanics, a recent challenge of interest is to have advanced numerical methodologies for constructing robust

*Address all correspondence to this author. computational models in order to efficiently predict the mechanical behavior of structures. In numerous industrial applications, the effects of geometrical nonlinearities induced by large strains and by large displacements have to be taken into account in the numerical modeling. For instance, such nonlinear mechanical behavior is exhibited in aeronautics by helicopter rotating blades $[1,2]$ or in automotive or aerospatial industries by slender beams or thin shells [3-5]. In the context of complex structures, large finite element computational models are needed. Given the numerical difficulties inherent to the complexity of such computational models, many recent efforts have focused on the construction of reduced order models in this nonlinear context $[6,7]$. In particular, the STEP procedure $[8,9]$ has been developed in order to explicitly construct all the linear, quadratic, cubic stiffness terms related to reduced nonlinear models. The methodology is based on the smart use of an industrial finite element code for which no further numerical developments are needed. It only requires a series of basic nonlinear numerical calculations with judiciously prescribed displacements taken as a linear combination of given basis vectors.

Moreover, deterministic nonlinear computational models are in general not sufficient to accurately predict the mechanical behavior of such complex structures. The uncertainties have then to be taken into account in the computational models by using prob- 
abilistic models as soon as the probability theory can be used. Let us recall that there exist two classes of uncertainties: (1) the system parameter uncertainties result from the variability of the parameters of the computational model induced for instance by the manufacturing process, (2) the model uncertainties are the features of the mechanical system not captured by the computational model, e.g. the introduction of reduced kinematics in the numerical modeling. Parametric probabilistic approaches are particularly adapted to take into account system parameter uncertainties as shown in $[10,11]$ is the context of the post-buckling of cylindrical shells. This last decade, the nonparametric probabilistic approach adapted to the modeling of both model uncertainties and system parameter uncertainties has been introduced in $[12,13]$ for the linear case and has been extended more recently in $[9,14-16]$ in the context of geometrical nonlinearities.

In the present paper, a direct procedure is proposed in the context of geometrical nonlinear structural mechanical systems. In this methodology, the explicit construction of the uncertain nonlinear computational model is proposed for any type of structure modeled with three-dimensional solid finite elements.

In the first Section, the equations of the geometrical nonlinear problem are written in the context of a total Lagrangian formulation. The second Section is devoted to the construction of a mean reduced nonlinear computational model required by the implementation of the nonparametric probabilistic approach. This mean reduced nonlinear computational model is obtained using the Proper Orthogonal Decomposition method known to be particularly efficient in nonlinear cases [6]. The POD-basis is then easily deduced from a reference solution taken as the deterministic response of the structure. The mean reduced nonlinear computational model, which results from the projection of all the linear, quadratic and cubic stiffness terms on this POD-basis is then explicitly constructed in the context of three-dimensional solid finite elements. The third Section is devoted to the construction of the uncertain nonlinear computational model using the nonparametric probabilistic approach. Such nonparametric probabilistic approach is based on the construction of a probability model for random matrices with values in the set of symmetric positive-definite matrices whose mean value is deduced from the mean reduced computational model. Let us recall that the nonlinear quadratic stiffness term of the mean reduced nonlinear computational model is defined as the sum of three nonlinear terms. In the present geometrical nonlinear context, the nonparametric probabilistic model is implemented from a deterministic symmetric positive-definite matrix whose components are notably described by each of these three nonlinear terms. Note that its explicit construction is then needed. In the fourth Section, the procedure for the robust identification of the uncertain nonlinear computational model with respect to available experimental responses is carried out $[17,18]$. Finally, the fifth Section is devoted to a numerical example in order to demonstrate the efficiency of the proposed methodology.

\section{FORMULATION OF THE GEOMETRIC NONLINEAR PROBLEM}

The structure under consideration is made up of a linear elastic material and is assumed to undergo large deformations induced by geometrical nonlinearity. Let $\Omega$ be the threedimensional bounded domain of the physical space $\mathbb{R}^{3}$ corresponding to the reference configuration taken as a natural state without prestress. The boundary $\partial \Omega$ is such that $\partial \Omega=\Gamma \bigcup \Sigma$ with $\Gamma \bigcap \Sigma=\emptyset$ and the external unit normal to boundary $\partial \Omega$ is denoted as $\mathbf{n}$. The boundary part $\Gamma$ corresponds to the fixed part of the structure whereas the boundary part $\Sigma$ is subjected to an external surface force field. A total Lagrangian formulation is chosen. Consequently, the mechanical equations are written with respect to the reference configuration. Let $\mathbf{x}$ be the position of a point belonging to domain $\Omega$. The displacement field expressed with respect to the reference configuration is denoted as $\mathbf{u}(\mathbf{x})$. It should be noted that the surface force field $\mathbf{G}(\mathbf{x})$ acting on boundary $\Sigma$ and that the body force field $\mathbf{g}(\mathbf{x})$ acting on domain $\Omega$ corresponds to the Lagrangian transport of the physical surface force field and to the physical body force field applied on the deformed configuration into the reference configuration. Let $\mathcal{C}$ be the admissible space defined by

$$
\mathcal{C}=\{\mathbf{v} \in \Omega, \mathbf{v} \text { sufficiently regular }, \mathbf{v}=\mathbf{0} \text { on } \Gamma\}
$$

The weak formulation of the geometric nonlinear static boundary problem consists in finding the unknown displacement field $\mathbf{u}$ of admissible space $\mathcal{C}$ such that, for any admissible displacement field $\mathbf{v} \in \mathcal{C}$

$$
\int_{\Omega} v_{i, k} F_{i j} S_{j k} d \mathbf{x}=\int_{\Omega} v_{i} g_{i} d \mathbf{x}+\int_{\Sigma} v_{i} G_{i} d \mathbf{s}
$$

in which $\mathbb{E}$ is the deformation gradient tensor whose components $F_{i j}$ are defined by

$$
F_{i j}=u_{i, j}+\delta_{i j}
$$

in which $\delta_{i j}$ is the Kronecker symbol such that $\delta_{i j}=1$ if $i=j$ and $\delta_{i j}=0$ if not, and where $\mathbb{S}$ is the second Piola-Kirchoff symmetric stress tensor defined for any elastic material by the linear relation

$$
S_{i j}=a_{i j k l} E_{k l}
$$

In Eq. (4), the fourth order elasticity tensor a satisfies the classical symmetry and positive definiteness properties. The Green strain tensor $\mathbb{E}$ is then written as the sum of a linear term and of a nonlinear term such that

$$
E_{i j}=\varepsilon_{i j}+\eta_{i j}
$$


in which

$$
\varepsilon_{i j}=\frac{1}{2}\left(u_{i, j}+u_{j, i}\right) \quad \text { and } \quad \eta_{i j}=\frac{1}{2} u_{s, i} u_{s, j}
$$

The weak formulation Eq. (2) is reformulated as finding the unknown displacement field $\mathbf{u}$ of admissible space $\mathcal{C}$ such that, for any admissible displacement field $\mathbf{v} \in \mathcal{C}$ we have

$$
k^{(1)}(\mathbf{u}, \mathbf{v})+k^{(2)}(\mathbf{u}, \mathbf{u}, \mathbf{v})+k^{(3)}(\mathbf{u}, \mathbf{u}, \mathbf{u}, \mathbf{v})=l(\mathbf{v})
$$

in which the multilinear forms $l(\mathbf{v}), k^{(1)}(\mathbf{u}, \mathbf{v}), k^{(2)}(\mathbf{u}, \mathbf{u}, \mathbf{v})$ and $k^{(3)}(\mathbf{u}, \mathbf{u}, \mathbf{u}, \mathbf{v})$ are defined for all $\mathbf{u}, \mathbf{v} \in \mathcal{C}$ by

$$
\begin{aligned}
& l(\mathbf{v})=\int_{\Omega} v_{i} g_{i} d \mathbf{x}+\int_{\Sigma} v_{i} G_{i} d \mathbf{s}, \\
& k^{(1)}(\mathbf{u}, \mathbf{v})=\int_{\Omega} a_{j k l m} \varepsilon_{l m}(\mathbf{u}) \varepsilon_{j k}(\mathbf{v}) d \mathbf{x}, \\
& k^{(2)}(\mathbf{u}, \mathbf{u}, \mathbf{v})=\int_{\Omega} a_{j k l m} \eta_{l m}(\mathbf{u}) \varepsilon_{j k}(\mathbf{v}) d \mathbf{x}+ \\
& \int_{\Omega} a_{j k l m} u_{s, j} v_{s, k} \varepsilon_{l m}(\mathbf{u}) d \mathbf{x} \\
& k^{(3)}(\mathbf{u}, \mathbf{u}, \mathbf{u}, \mathbf{v})=\int_{\Omega} a_{j k l m} u_{s, j} v_{s, k} \eta_{l m}(\mathbf{u}) d \mathbf{x} .
\end{aligned}
$$

\section{CONSTRUCTION OF A MEAN REDUCED COMPUTA- TIONAL MODEL FOR THE GEOMETRICAL NONLIN- EAR PROBLEM}

This Section concerns the construction of the mean reduced computational model adapted to the geometrical nonlinear context. The methodology is based on the explicit construction of each term constituting this mean reduced nonlinear computational model. It is recalled that such mean reduced nonlinear computational model is required by the nonparametric probabilistic modeling of uncertainties. First, the general equations yielding the mean reduced nonlinear computational model are written for any given projection basis. Then, the Proper Orthogonal Decomposition method is used for constructing the projection basis in the context of large finite elements systems [6, 19]. This POD-basis is deduced from a reference calculation using a finite element code. Finally, each linear, quadratic and cubic stiffness component constituting the mean reduced nonlinear computational model is explicitly constructed in the context of three-dimensional solid finite elements.
General equations of the mean reduced model

Let $\varphi^{\alpha}(\mathbf{x}), \alpha=\{1, \ldots, N\}$, be a given set of basis functions such that

$$
\mathbf{u}(\mathbf{x})=\sum_{\beta=1}^{N} \varphi^{\beta}(\mathbf{x}) q_{\beta},
$$

in which the $\mathbb{R}^{N}$-vector $\mathbf{q}=\left(q_{1}, \ldots, q_{N}\right)$ is the vector of the generalized coordinates. Let $\mathbf{v}(\mathbf{x})$ be a test function such that

$$
\mathbf{v}(\mathbf{x})=\varphi_{\alpha}(\mathbf{x}) q_{\alpha}
$$

Replacing Eq.(13) in Eq.(7) yields the following set of nonlinear equations

$$
\mathcal{K}_{\alpha \beta}^{(1)} q_{\beta}+\mathcal{K}_{\alpha \beta \gamma}^{(2)} q_{\beta} q_{\gamma}+\mathcal{K}_{\alpha \beta \gamma \delta}^{(3)} q_{\beta} q_{\gamma} q_{\delta}=\mathcal{F}_{\alpha}
$$

in which

$$
\mathcal{K}_{\alpha \beta \gamma}^{(2)}=\frac{1}{2}\left(\hat{\mathcal{K}}_{\alpha \beta \gamma}^{(2)}+\hat{\mathcal{K}}_{\beta \gamma \alpha}^{(2)}+\hat{\mathcal{K}}_{\gamma \alpha \beta}^{(2)}\right)
$$

and where the expressions of $\mathcal{K}_{\alpha \beta}^{(1)}, \hat{\mathcal{K}}_{\alpha \beta \gamma}^{(2)}$ and $\hat{\mathcal{K}}_{\alpha \beta \gamma \delta}^{(3)}$ and its symmetry properties can be found in [9].

\section{Numerical construction of the reduced order basis us- ing Proper Orthogonal Decomposition}

The set of basis vectors used for constructing the mean reduced nonlinear computational model is obtained with the Proper Orthogonal Decomposition method which is known to be efficient for nonlinear cases. The determination of this basis necessarily requires a reference response. Indeed such basis is defined by the eigenvalue problem of the spatial correlation operator related to the displacement field of this reference response. It should be noted that this basis does not only depend on the operators of the computational model but also strongly depends on the external applied loads used for exciting the structure. Below, the numerical construction of the POD-basis is summarized in the context of the finite element method. The finite element discretization of Eq. (7) can be written as

$$
\left[K^{(1)}\right] \mathbf{u}+\mathbf{f}^{N L}(\mathbf{u})=\mathbf{f}
$$

in which the $\mathbb{R}^{n}$-vector $\mathbf{u}$ is the vector of the unknown displacements. In Eq. (16), the $(n \times n)$ symmetric positive definite matrix $\left[K^{(1)}\right]$ is the linear finite element stiffness matrix, the $\mathbb{R}^{n}$-vector 
$\mathbf{f}^{N L}(\mathbf{u})$ is the vector of the restoring forces induced by the geometrical nonlinear effects and the $\mathbb{R}^{n}$-vector $\mathbf{f}$ is the vector of the external applied loads. It should be noted that there are specific numerical algorithms for solving this nonlinear equation (see for instance [20]) which are particularly efficient as the curvature of the nonlinear response changes (see for instance [21] for algorithms based on arc-length methods or [22] for algorithms based on asymptotic methods).

Let $s_{j} \in[0,1], j \in\{1, \ldots, p\}$ with $s_{j}<s_{j+1}$ be the scalar denoting the incremental weight number $j$ of the external load vector $\mathbf{f}$. The $(n \times p)$ real matrix $[V]$ is then introduced as

$$
[V]_{i j}=u_{i}\left(s_{j}\right) \sqrt{\Delta s_{j}} \quad, \quad \Delta s_{j}=s_{j}-s_{j-1} \text { with } s_{0}=0 .
$$

The spatial correlation matrix related to the nonlinear reference response is defined by the symmetric positive-definite $(n \times n)$ real matrix $[A]$ such that

$$
[A]=[V][V]^{T}
$$

The POD-basis is then obtained in solving the following eigenvalue problem

$$
[A][\Phi]=[\Phi][\Lambda]
$$

in which $[\Lambda]$ is the diagonal matrix whose components are the eigenvalues ordered by decreasing values and where $[\Phi]$ is the modal matrix whose columns are the POD-basis vectors. It should be noted that such numerical construction can not be carried out as the dimension $n$ of the system increases. The singular value decomposition of matrix $[V]$ is written as

$$
[V]=[B][S][C]
$$

in which $[S]=[\Lambda]^{1 / 2}$ and where the columns of the $(n \times n)$ real matrices $[B]$ and $[C]$ are the left and right singular vectors related to the corresponding singular values. As a consequence, it can be shown that

$$
[\Phi]=[B] \text { and }[S]^{2}=[\Lambda]
$$

Let $[B]^{N}$ be the $(n \times N)$ matrix issued from the truncation of matrix $[B]$ with respect to the $N$ largest singular values. Matrix $[B]^{N}$ can easily be computed by using an adapted algorithm for truncated single value decomposition [23].

\section{Construction of the mean reduced nonlinear computa- tional model}

In this subsection, the mean reduced nonlinear computational model is explicitly constructed from the knowledge of the POD-basis. The construction is carried out in the context of the three-dimensional finite element method. The finite elements used are isoparametric solid finite elements with 8 nodes and the numerical integration is carried out with $r$ Gauss integration points.

Let $[D]$ be the $(6 \times 6)$ real matrix which represents the usual Hooke matrix related to the fourth-order elasticity tensor. For the considered isoparametric finite element, the displacement field $\tilde{\mathbf{u}}(\mathbf{y})$ with $\mathbf{y} \in[-1,1]^{3}$, is defined by

$$
\tilde{\mathbf{u}}(\mathbf{y})=[N(\mathbf{y})] \tilde{\mathbf{u}}
$$

in which the $(3 \times 24)$ real matrix $[N(\mathbf{y})]$ defines the interpolation functions and where the $\mathbb{R}^{24}$-vector $\tilde{\mathbf{u}}$ is made up of the degrees of freedom of the finite element. Let $\mathcal{I}$ be the set of indices defined by $\mathcal{I}=\{(i, j) \in\{(11),(22),(33),(12),(13),(23)\}\}$ and corresponding with the set $\mathfrak{I}=\{1,2,3,4,5,6\}$. From Eq. (22), it can be deduced that

$$
\begin{aligned}
\varepsilon_{i j}(\tilde{\mathbf{u}})\left(1-\delta_{i j}\right)+\varepsilon_{j i}(\tilde{\mathbf{u}}) & =[B(\mathbf{y})]_{I k} \tilde{u}_{k}(i, j) \in \mathcal{I}, I \in \mathfrak{I} \\
\tilde{u}_{s, l}(\mathbf{y}) \tilde{u}_{s, m}(\mathbf{y}) & =\tilde{\mathbf{u}}^{T}\left[C_{l}(\mathbf{y})\right]^{T}\left[C_{m}(\mathbf{y})\right] \tilde{\mathbf{u}}
\end{aligned}
$$

in which $[B(\mathbf{y})]$ and $\left[C_{m}(\mathbf{y})\right]$ are the $(6 \times 24)$ and $(3 \times 24)$ real matrices whose components are obtained by the calculation of partial derivatives of the interpolation functions contained in ma$\operatorname{trix}[N(\mathbf{y})]$.

The first step consists in calculating for each finite element the elementary contributions of the linear, quadratic and cubic internal forces projected on the POD-basis. Then, for a given finite element, let $\tilde{\mathbf{f}}^{(1)}\left(\tilde{\varphi}^{\beta}\right), \tilde{\mathbf{f}}^{(2)}\left(\tilde{\varphi}^{\beta}, \tilde{\varphi}^{\gamma}\right)$ and $\tilde{\mathbf{f}}^{(3)}\left(\tilde{\varphi}^{\beta}, \tilde{\varphi}^{\gamma}, \tilde{\varphi}^{\delta}\right)$ be the $\mathbb{R}^{24}$-vectors constituted of the internal forces, (1) induced by the POD-basis vector $\varphi^{\beta}$ and related to the linear stiffness term, (2) induced by the POD-basis vectors $\varphi^{\beta}, \varphi^{\gamma}$ and related to the quadratic stiffness term, induced by the POD-basis vector $\varphi^{\beta}, \varphi^{\gamma}, \varphi^{\delta}$ and related to the cubic stiffness term. All details concerning the explicit construction of these terms can be found in $[15,16]$. In a second step, for each type of stiffness, we proceed with the assembly of each of these elementary contributions. We then denote by $\mathbf{f}^{(1)}\left(\varphi^{\beta}\right), \mathbf{f}^{(2)}\left(\varphi^{\beta}, \varphi^{\gamma}\right)$ and $\mathbf{f}^{(3)}\left(\varphi^{\beta}, \varphi^{\gamma}, \varphi^{\delta}\right)$ the $\mathbb{R}^{n}$-vectors of these internal loads. The mean reduced nonlinear computational model is then described by

$$
\begin{aligned}
\mathcal{K}_{\alpha \beta}^{(1)} & =\varphi^{\alpha, T} \mathbf{f}^{(1)}\left(\varphi^{\beta}\right) \\
\hat{\mathcal{K}}_{\alpha \beta \gamma}^{(2)} & =\varphi^{\alpha, T} \mathbf{f}^{(2)}\left(\varphi^{\beta}, \varphi^{\gamma}\right) \\
\mathcal{K}_{\alpha \beta \gamma \delta}^{(3)} & =\varphi^{\alpha, T} \mathbf{f}^{(3)}\left(\varphi^{\beta}, \varphi^{\gamma}, \varphi^{\delta}\right)
\end{aligned}
$$


The quadratic stiffness contribution $\mathcal{K}_{\alpha \beta \gamma}^{(2)}$ of the mean reduced nonlinear computational model is then build from Eq. (15). It should be noted that the $\mathcal{K}_{\alpha \beta}^{(1)}, \hat{\mathcal{K}}_{\alpha \beta \gamma}^{(2)}$ and $\mathcal{K}_{\alpha \beta \gamma \delta}^{(3)}$ contributions have to be explicitly known for constructing the uncertain nonlinear computational model in the general case of complex structures.

\section{NONPARAMETRIC STOCHASTIC MODELING OF UN- CERTAINTIES}

In this Section, it is assumed that the mean reduced nonlinear computational model contains both system parameter uncertainties and model uncertainties which justifies the use of the nonparametric probabilistic approach for modeling these uncertainties in the computational model. The main idea of the nonparametric probabilistic approach consists in replacing each of the matrices of a given mean reduced computational model by a random matrix whose probability model is constructed from the maximum entropy principle using the available information $[12,13]$. In the usual linear case, the random matrices issued from the mechanical system are with values in the set of the symmetric positive-definite matrices. In the present geometrical nonlinear context, the nonlinear equations involve nonlinear operators. In this case, we then introduce the matrix $[\mathcal{K}][9]$ as the real $(P \times P)$ matrix with $P=N(N+1)$ defined by

$$
[\mathcal{K}]=\left[\begin{array}{cc}
{\left[\mathcal{K}^{(1)}\right]} & {\left[\hat{\mathcal{K}}^{(2)}\right]} \\
{\left[\hat{\mathcal{K}}^{(2)}\right]^{T}} & 2\left[\mathcal{K}^{(3)}\right]
\end{array}\right]
$$

in which $\left[\hat{\mathcal{K}}^{(2)}\right]$ and $\left[\mathcal{K}^{(3)}\right]$ are respectively the $\left(N \times N^{2}\right)$ and $\left(N^{2} \times N^{2}\right)$ real matrices resulting from the following reshaping operation defined by

$$
\left[\hat{\mathcal{K}}^{(2)}\right]_{\alpha J}=\hat{\mathcal{K}}_{\alpha \beta \gamma}^{(2)} \quad, \quad \text { with } J=(\beta-1) N+\gamma \quad
$$

$\left[\mathcal{K}^{(3)}\right]_{I J}=\mathcal{K}_{\alpha \beta \gamma \delta}^{(3)}$ with $I=(\alpha-1) N+\beta$ and $J=(\gamma-1) N+\delta$.

It is shown in [9] that matrix $[\mathcal{K}]$ is a symmetric positive-definite matrix. Consequently, the nonparametric probabilistic approach can easily be adapted to the geometrically nonlinear context as follows. The mean reduced matrix $[\mathcal{K}]$ is replaced by the random matrix $[\mathcal{K}]$ such that $\mathcal{E}\{[\mathcal{K}]\}=[\mathcal{K}]$ in which $\mathcal{E}$ is the mathematical expectation. The random matrix $[\mathcal{K}]$ is then written as $[\mathcal{K}]=\left[L_{K}\right]^{T}\left[\mathbf{G}_{K}\right]\left[L_{K}\right]$ in which $\left[L_{K}\right]$ is a $(P \times P)$ real upper matrix such that $[\mathcal{K}]=\left[L_{K}\right]^{T}\left[L_{K}\right]$ and where $\left[\mathbf{G}_{K}\right]$ is a full random matrix with value in the set of all the positive-definite symmetric $(P \times P)$ matrices. The probability model of random matrix $\left[\mathbf{G}_{K}\right]$ is constructed by using the maximum entropy principle with the available information. All details concerning the construction of this probability model can be found in $[12,13]$. The dispersion of random matrix $\left[\mathbf{G}_{K}\right]$ is controlled by one real positive parameter $\delta \in \mathcal{D}$ called the dispersion parameter. In addition, there exists an algebraic representation of this random matrix useful to the Monte Carlo numerical simulation. From random matrix $[\mathcal{K}]$ the random linear, quadratic and stiffness terms $\mathcal{K}_{\alpha \beta}^{(1)}, \hat{\mathcal{K}}_{\alpha \beta \gamma}^{(2)}$ and $\mathcal{K}_{\alpha \beta \gamma \delta}^{(3)}$ can easily be deduced. The random matrix model is then defined by

$$
\mathbf{U}=\left[\Phi^{N}\right] \mathbf{Q}
$$

in which $\mathbf{Q}=\left(\mathbf{Q}_{1}, \ldots, \mathbf{Q}_{N}\right)$ is the $\mathbb{R}^{N}$-valued vector of the random generalized coordinates solution of the random equation

$$
\mathcal{K}_{\alpha \beta}^{(1)} \mathbf{Q}_{\beta}+\mathcal{K}_{\alpha \beta \gamma}^{(2)} \mathbf{Q}_{\beta} \mathbf{Q}_{\gamma}+\mathcal{K}_{\alpha \beta \gamma \delta}^{(3)} \mathbf{Q}_{\beta} \mathbf{Q}_{\gamma} \mathbf{Q}_{\delta}=\mathcal{F}_{\alpha}
$$

with

$$
\mathcal{K}_{\alpha \beta \gamma}^{(2)}=\frac{1}{2}\left(\hat{\mathcal{K}}_{\alpha \beta \gamma}^{(2)}+\hat{\mathcal{K}}_{\beta \gamma \alpha}^{(2)}+\hat{\mathcal{K}}_{\gamma \alpha \beta}^{(2)}\right)
$$

\section{IDENTIFICATION OF THE UNCERTAIN NONLINEAR COMPUTATIONAL MODEL}

In this Section, the identification of the uncertain nonlinear computational model from experimental data is formulated. It is assumed that the mean reduced nonlinear computational is known and that the identification focuses on the parameter $\delta$ controlling the uncertainty level in the uncertain computational model. This robust updating problem consists then in minimizing a cost function with respect to parameter $\delta$. The formulation of the optimization problem requires the definition of a cost function relevant to the uncertain nonlinear computational model and to the experimental data. It is proposed here to introduce penalty terms only in areas for which the experimental data is not within the confidence region constructed with the uncertain nonlinear computational model. It is assumed that a collection of $n_{\text {exp }}$ experimental responses are available at $n_{o b s}$ spatial locations. We then denote as $U_{j}^{\text {exp }}\left(s, \theta_{k}\right)$ the experimental response number $k$ at dof number $j$ as a function of the load increment $s$. The corresponding observation issued from the uncertain computational model is denoted as $U_{j}(\delta, s)$ and is a function of the parameter $\delta$ to be updated. Let $U_{j}^{+}(\delta, s)$ (resp. $\left.U_{j}^{-}(\delta, s)\right)$ and $U_{j}^{\text {exp },+}(s)$ (resp. $U_{j}^{\text {exp },-}(s)$ ) be the upper (resp. lower) envelope of the confidence region of observation $U_{j}(\delta, s)$ obtained with a probability level $\alpha=0.95$ and the upper (resp. lower) envelope of experiments 
$U_{j}^{e x p}(s)$. The cost function $j(\delta)$ is then defined by

$$
j(\delta)=\left\|\boldsymbol{\Delta}^{+}(\boldsymbol{\delta}, \cdot)\right\|_{\mathbb{B}}^{2}+\left\|\boldsymbol{\Delta}^{-}(\delta, \cdot)\right\|_{\mathbb{B}}^{2},
$$

in which $\|\cdot\|_{\mathbb{B}}$ is the $\mathcal{L}^{2}$ norm over the load incremental band $\mathbb{B}=[0, b]$ and where $\boldsymbol{\Delta}^{+}(\boldsymbol{\delta}, s)$ and $\boldsymbol{\Delta}^{-}(\boldsymbol{\delta}, s)$ are the $\mathbb{R}^{n_{o b s}}$ vectors whose component number $j$ is defined by

$$
\begin{aligned}
\Delta_{j}^{+}(\delta, s)= & \left\{U_{j}^{+}(\delta, s)-U_{j}^{e x p,+}(s)\right\} \times \\
& \left\{1-H\left(U_{j}^{+}(\delta, s)-U_{j}^{\text {exp },+}(s)\right)\right\}, \\
\Delta_{j}^{-}(\delta, s)= & \left\{U_{j}^{-}(\delta, s)-U_{j}^{e x p,-}(s)\right\} \times \\
& \left.\left\{H\left(U_{j}^{-}(\delta, s)-U_{j}^{\text {exp },+}(s)\right)\right)\right\},
\end{aligned}
$$

where $x \mapsto H(x)$ is the Heaviside function. The identification of the uncertain nonlinear computational model consists then in solving the optimization problem

$$
\text { find } \delta^{o p t} \in \mathcal{D} \text { such that } j\left(\delta^{o p t}\right) \leq j(\delta) \quad, \quad \forall \delta \in \mathcal{D} .
$$

\section{NUMERICAL APPLICATION}

The objective of this application is to show the efficiency of the presented methodology. The application is a threedimensional linear elastostatic problem in the geometrically nonlinear context. The material is chosen to be homogeneous and isotropic. The extension to the nonhomogeneous case and to the anisotropic case is straightforward. A preliminary calculation is carried out with a non-linear finite element code in order to get the reference solution from which the POD basis is calculated. The stochastic nonlinear reduced-order computational model is then constructed as a function of identification parameter $\delta$.

Note that the simulated experiments have been obtained by numerical simulations for a family of structures around the mean structure. Specifically, the geometrical characteristics of each structure of the family are modified with respect to those of the mean structure. Moreover, the material characteristics of these structures are inhomogenous at the contrary of the mean structure. Consequently, the mean computational model can never reproduce the simulated experiments which justifies the use of a stochastic nonlinear reduced-order computational model.

\section{Mean finite element model}

The three-dimensional bounded domain $\Omega$ is a slender rectangular domain defined in a Cartesian system $\left(0, \mathbf{e}_{1}, \mathbf{e}_{2}, \mathbf{e}_{3}\right)$ such that $\Omega=\{] 0, l[\times] 0, b[\times] 0, h[\}$ with $l=10 \mathrm{~m}, b=0.8 \mathrm{~m}, h=$ $0.4 \mathrm{~m}$. Let $\Gamma_{0}$ and $\Gamma_{1}$ be the boundaries described as $\Gamma_{0}=$ $\left\{\mathbf{x} ; x_{1}=0\right\}$ and $\Gamma_{1}=\left\{\mathbf{x} ; x_{1}=L\right\}$. The structure is assumed to be fixed on this boundary so that we have a Dirichlet condition on $\Gamma_{0} \bigcup \Gamma_{1}$. The structure is free on boundary $\partial \Omega \backslash\left\{\Gamma_{0} \bigcup \bigcup \Gamma_{1}\right\}$. The structure is subjected to external surface loads applied along directions $\mathbf{e}_{1}, \mathbf{e}_{2}$ and $\mathbf{e}_{3}$ in the middle section defined by $x_{1}=$ $0.5 \mathrm{~L}$. The Young modulus, the Poisson coefficient and the mass density of the homogeneous and isotropic linear elastic material are $E=2.10^{11} \mathrm{~N} . \mathrm{m}^{-2}, v=0.3$ and $\rho=8200 \mathrm{Kg} . \mathrm{m}^{-3}$. The finite element model is a regular mesh of 765 nodes and $50 \times 4 \times 2=400$ finite elements constituted of 8 -nodes solid elements with $r=8$ Gauss integration points. Therefore, the mean computational model has $n=2205$ degrees of freedom (see Fig. 1).

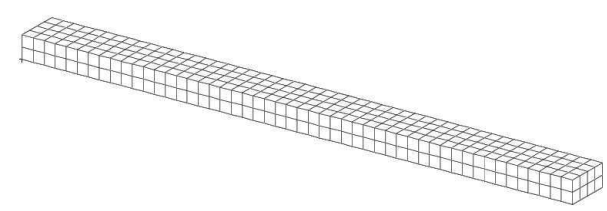

$$
L_{x}^{2}
$$

FIGURE 1. FINITE ELEMENT MODEL

The discretization of the external loads yields point loads applied to the nodes of the middle section along the direction $\mathbf{e}_{1}$, $\mathbf{e}_{2}$ and $\mathbf{e}_{3}$ with intensity $100,000,000 \mathrm{~N}$. Furthermore, the gravity load is also taken into account. In order to simulate the nonlinear mechanical response, the static nonlinear calculations are carried out by solving Eq. (16) using a nonlinear finite element code with an algorithm based on the arc-length method. The displacement field is calculated using $n_{t}=60$ load increments. Fig. 2 shows the deformed structure for the nonlinear static case.

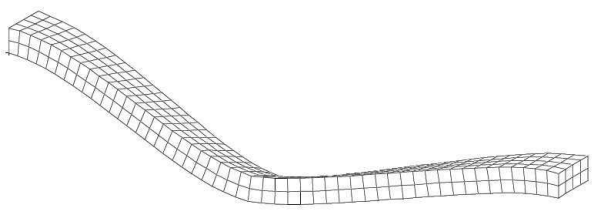

$K^{r}$

FIGURE 2. DEFORMED STRUCTURE 


\section{Construction of the experimental data basis}

In this numerical application, $n_{\exp }=9$ simulated experiments related to the static nonlinear response are calculated. The simulated experiments are observed at $n_{o b s}=2$ observation points corresponding to the DOFs located at the bottom of the middle section in the directions $\mathbf{e}_{2}$ and $\mathbf{e}_{3}$. The simulated static nonlinear responses are denoted by $u_{o b s}^{e x p}(s)$, for $k$ in $\left\{1, \ldots, n_{\exp }\right\}$. The corresponding quantity defined for the mean nonlinear computational model is denoted by $u_{o b s}(s)$. The simulated experiments are generated as follows. The geometrical parameters $l, b$ and $h$ are replaced by the random uniform variables $L, B$ and $H$ centered around geometrical parameters $l, b$ and $h$ with supports $[0.95 l, 1.05 l],[0.95 b, 1.05 b]$ and $[0.95 h, 1.05 h]$. Moreover, it is assumed that the Young modulus and the Poisson ratio are inhomogeneous with $10 \%$ of variation around their mean value. This is achieved by replacing the deterministic value $E(\operatorname{resp} v)$ by a stochastic field $E(\mathbf{x})(\operatorname{resp} v(\mathbf{x}))$ which is defined by

$$
\begin{gathered}
E(\mathbf{x})=E+\sum_{j=1}^{J} \xi_{j} \mathbf{b}_{j}(\mathbf{x}), \\
v(\mathbf{x})=v+\sum_{j=1}^{J} \xi_{j}^{\prime} \mathbf{b}_{j}(\mathbf{x}),
\end{gathered}
$$

in which $\xi_{1}, \ldots, \xi_{J}$ (resp. $\left.\xi_{1}^{\prime}, \ldots, \xi_{J}^{\prime}\right)$ are independent uniform random variables with zero mean and standard deviation $\sigma=$ $0.1 E / \sqrt{3}$ (resp. $\sigma=0.1 v / \sqrt{3}$ ) and where the functions $\mathbf{b}_{j}(\mathbf{x})$ are given smooth functions. For convenience, these smooth functions are taken as the spatial average over each element of the eigenvectors associated with the $J=12$ lowest eigenvalues of the usual generalized eigenvalue problem related to the linear dynamics.

Figures 3 and 4 compare the static nonlinear responses as a function of the incremental load $s$ for both mean nonlinear computational model and simulated experiments. Since the simulated experiments are scattered around the response calculated with the mean nonlinear computational model, it can be deduced that the use of a stochastic nonlinear computational model is justified.

\section{Construction of the POD basis}

The nonlinear response shown in Fig. 2 is then used for calculating the POD basis as described in Section 3.2. Let $\operatorname{Conv}_{\mathrm{POD}}(N)$ be the function defined by

$$
\operatorname{Conv}_{\mathrm{POD}}(N)=1-\frac{1}{\operatorname{tr}([A])} \sum_{j=1}^{N} \Lambda_{j}
$$

for which the calculation of $\operatorname{tr}([A])$ does not require the computation of matrix $[A]$. Fig. 5 shows the graph of the function

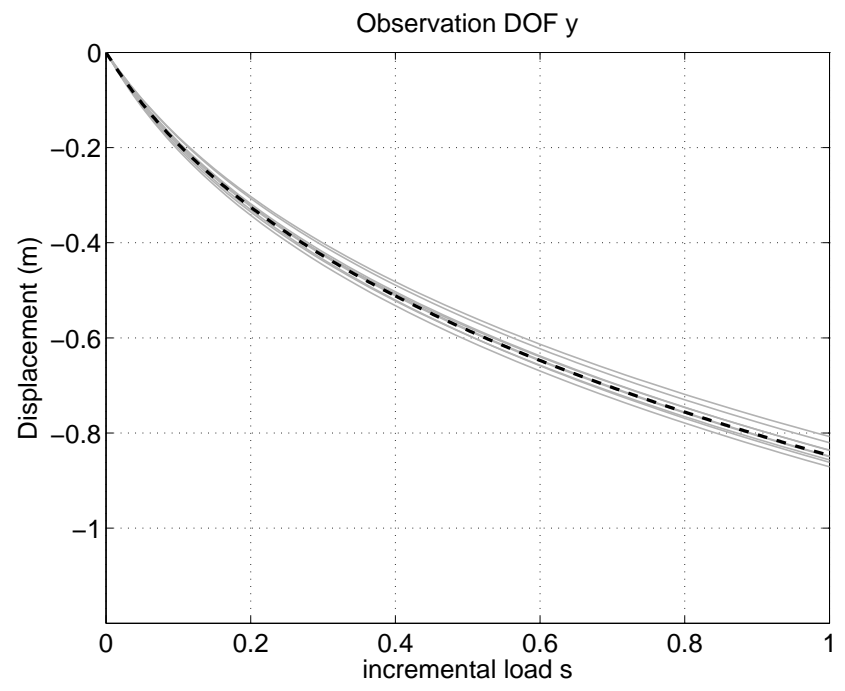

FIGURE 3. DISPLACEMENT RESPONSE AT OBSERVATION DOF $Y$ AS A FUNCTION OF THE INCREMENTAL LOAD $S$ : MEAN COMPUTATIONAL MODEL (THICK DASHED LINE), SIMULATED EXPERIMENTS (THIN GRAY LINES)

$N \mapsto \operatorname{Conv}_{\mathrm{POD}}(N)$ in a logarithmic scale. It can be seen that a good convergence is obtained for $N=10$. From now on, all numerical calculations are carried out with $N=12$. The mean nonlinear reduced-order computational model is then constructed and solved using the Crisfield algorithm [21] based on the arclength method.

\section{Experimental identification of the stochastic nonlinear reduced-order computational model}

The construction of the stochastic nonlinear reduced-order computational model using the nonparametric probabilistic approach is performed as explained in Section 4. A stochastic convergence analysis is then carried out to define the number $n_{s}$ of Monte Carlo realizations to be kept in the numerical simulation. Let $n_{s} \mapsto \operatorname{Conv}\left(n_{s}\right)$ be the function defined by

$$
\operatorname{Conv}\left(n_{s}\right)=\left\{\frac{1}{n_{s}} \sum_{j=1}^{n_{s}}\left\|\left|\mathbf{U}\left(\theta_{j}\right) \|\right|^{2}\right\}^{1 / 2}\right.
$$

in which $\left.\left.\left\|\mid \mathbf{U}\left(\theta_{j}\right)\right\|\left\|=\max _{s}\right\| \mathbf{U}\left(\theta_{j}, s\right)\right)\|, \quad\| \mathbf{U}\left(\theta_{j}, s\right)\right) \|^{2}=$ $\sum_{k=1}^{n} U_{k}^{2}\left(\theta_{j}, s\right)$ where $U_{k}\left(\theta_{j}, s\right)$ is the $j t h$ realization of the random response at DOF $k$ for a given load increment $s$. Figure 6 displays the graph $n_{s} \mapsto \operatorname{Conv}\left(n_{s}\right)$ obtained with a dispersion parameter $\delta=0.3$. Convergence is reached for $n_{s}=900$. The identification of $\delta$ is then carried out by constructing the non-differentiable 


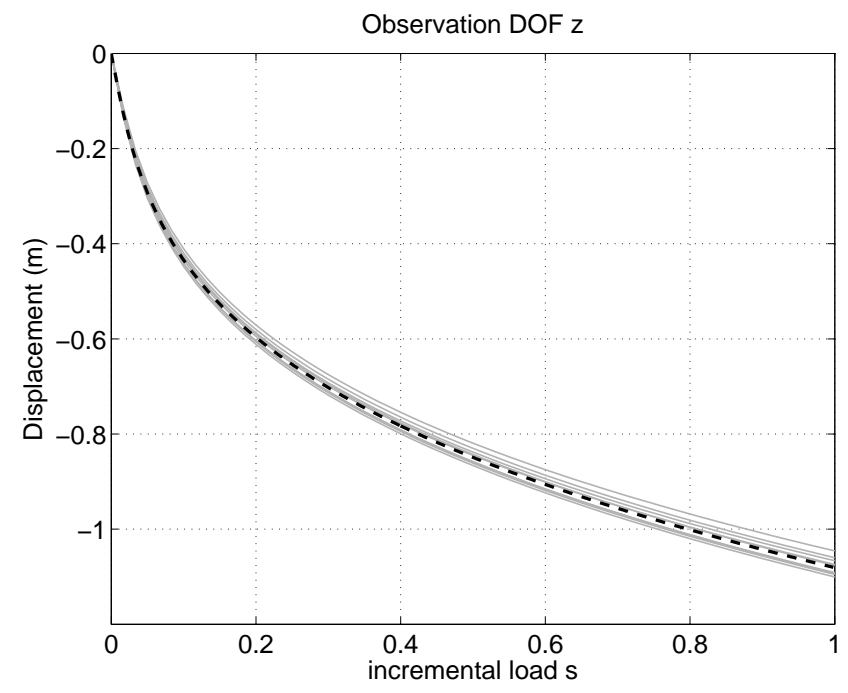

FIGURE 4. DISPLACEMENT RESPONSE OF OBSERVATION DOF $Z$ AS A FUNCTION OF THE INCREMENTAL LOAD $S$ : MEAN COMPUTATIONAL MODEL (THICK DASHED LINE), SIMULATED EXPERIMENTS (THIN GRAY LINES)

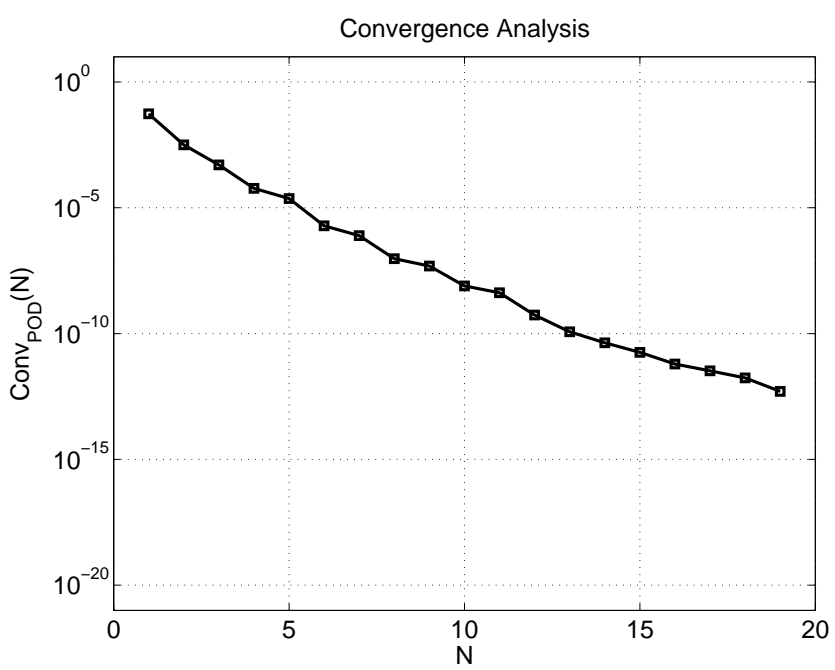

FIGURE 5. CONVERGENCE ANALYSIS : GRAPH OF $N \mapsto$ $\operatorname{Conv}_{\text {POD }}(N)$.

cost function $\delta \mapsto j(\delta)$ using the Monte Carlo numerical simulation. The cost function is a positive decreasing function of parameter $\delta$. As soon as the simulated experiments belong to the confidence region of the random observation, the cost function is equal to zero.

Figure 7 shows the graph of the cost function $\delta \mapsto j(\delta)$.

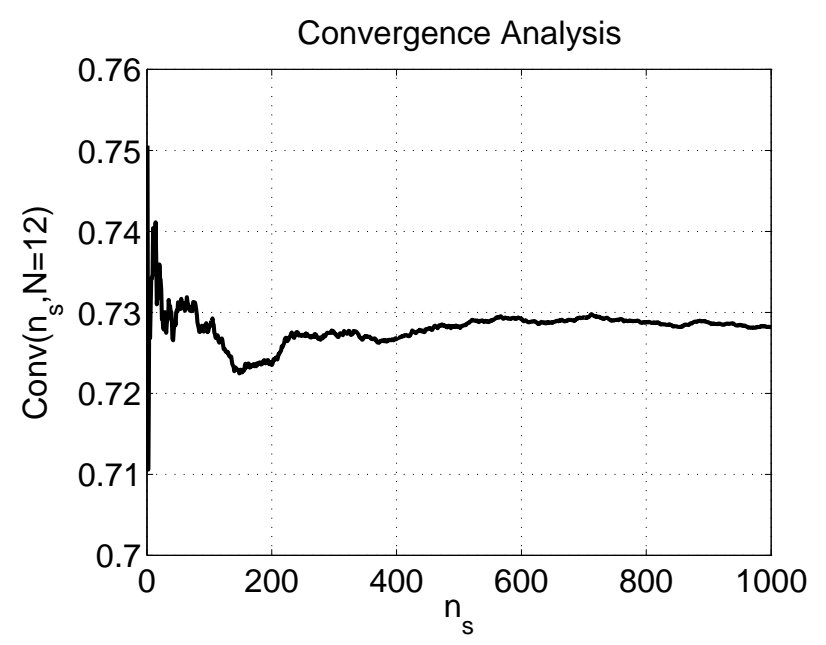

FIGURE 6. CONVERGENCE ANALYSIS : GRAPH OF $n_{s} \mapsto$ $\operatorname{Conv}\left(n_{s}\right)$.

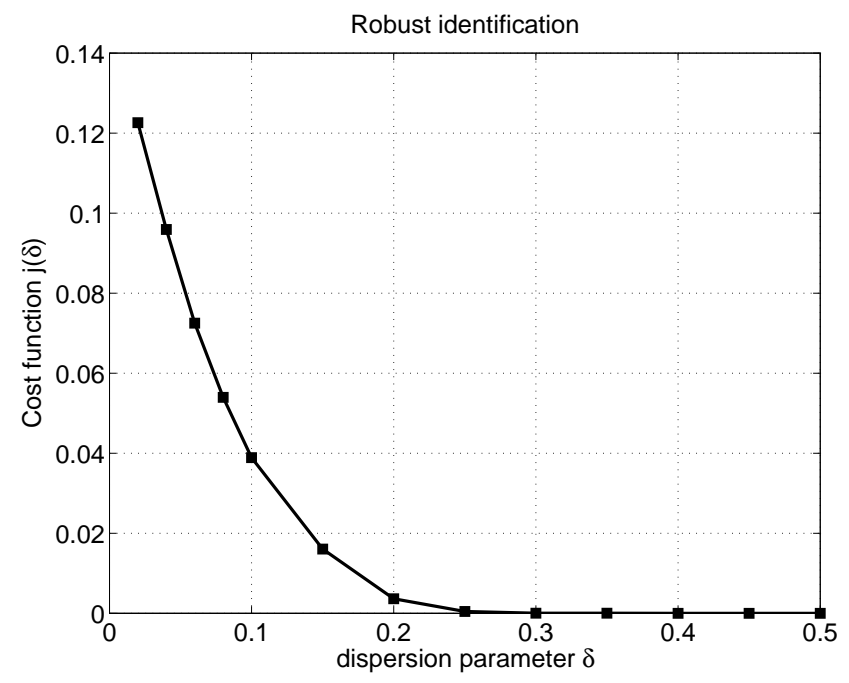

FIGURE 7. ROBUST IDENTIFICATION : GRAPH OF $\delta \mapsto j(\delta)$.

It can be seen that the optimal value is given by $\delta^{o p t}=0.3$. Figures 8 and 9 display the graph of the confidence region of the optimal random response $U_{o b s}\left(\delta^{o p t}, s\right)$ as a function of the load increment $s$ for both observations. It then can be seen that there is a good agreement between the optimal stochastic nonlinear reduced-order computational model and the simulated experiments. 


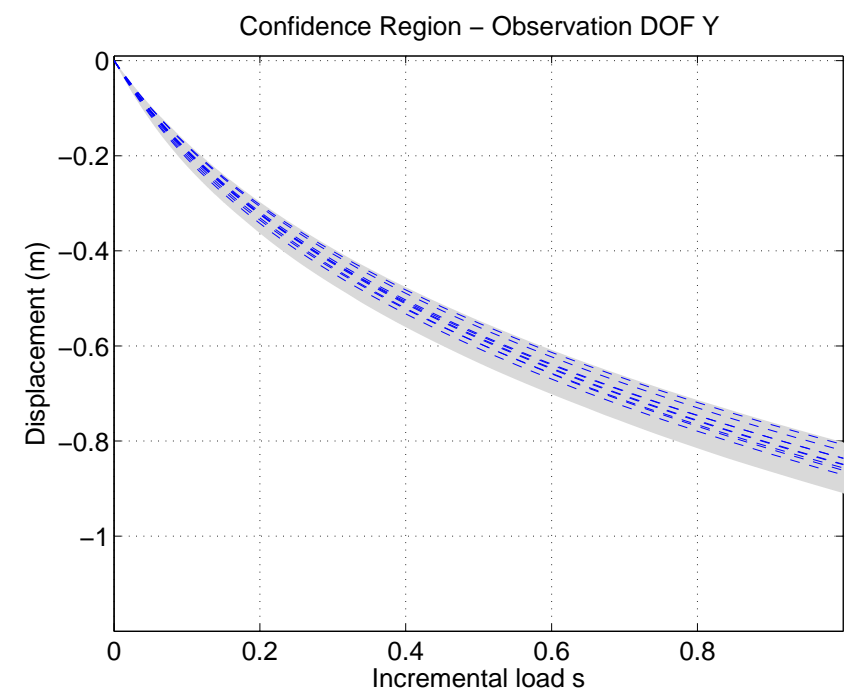

FIGURE 8. ROBUST IDENTIFICATION FOR OBSERVATION DOF Y : GRAPH OF THE EXPERIMENTAL DATA $s \mapsto u_{o b s}^{e x p, k}(s)$ (THIN DASHED LINES), GRAPH OF THE CONFIDENCE REGION OF THE RANDOM RESPONSE $s \mapsto U_{o b s}(s)$ (GREY REGION).

\section{Conclusion}

In the present paper, a methodology has been proposed for constructing a stochastic nonlinear reduced-order computational model for any three-dimensional structure with geometric nonlinearities and linear constitutive equation. The mean nonlinear reduced-order computational model is constructed by projection on the POD basis obtained from the mean nonlinear computational model. All the integrals involved in the weak formulation after projection on the POD basis are explicitly estimated using three-dimensional solid finite elements. The direct evaluation of the stiffness parameters of the mean nonlinear reduced-order model proposed and accomplished here guarantees the necessary properties (e.g. positive definiteness) of the model. It is further achievable for any three-dimensional finite element mean computational model. An application is presented and a methodology to perform the identification of the stochastic nonlinear reducedorder computational model using simulated experiments is proposed. If experimental data is available, the simulated experiments are then replaced by such data.

\section{REFERENCES}

[1] Pai, P., and Nayfeh, A., 1994. "A Fully Nonlinear-Theory of Curved And Twisted Composite Rotor Blades Accounting For Warpings and 3-Dimensional Stress Effects". International Journal of Solids and Structures, 31(9), MAY, pp. 1309-1340.

[2] Tang, D., and Dowell, E., 1996. "Nonlinear response of

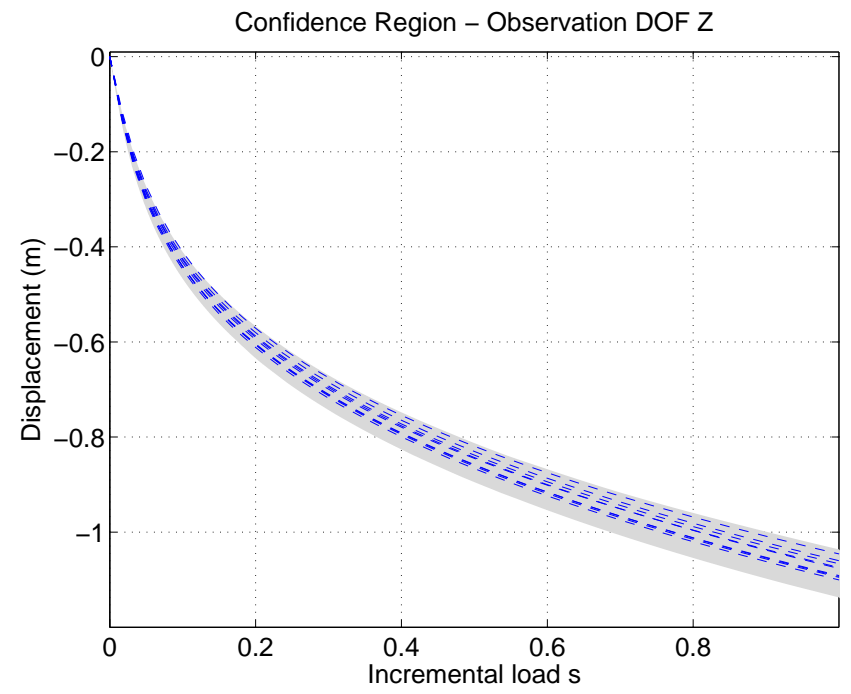

FIGURE 9. ROBUST IDENTIFICATION FOR OBSERVATION DOF Z: GRAPH OF THE EXPERIMENTAL DATA $s \mapsto u_{o b s}^{\text {exp }, k}(s)$ (THIN DASHED LINES), GRAPH OF THE CONFIDENCE REGION OF THE RANDOM RESPONSE $s \mapsto U_{o b s}(s)$ (GREY REGION).

a non-rotating rotor blade to a periodic gust". Journal of Fluids and Structures, 10(7), OCT, pp. 721-742.

[3] Hodges, D., Shang, X., and Cesnik, C., 1996. "Finite element solution of nonlinear intrinsic equations for curved composite beams". Journal of the American Helicopter Society, 41(4), OCT, pp. 313-321.

[4] Lindgaard, E., Lund, E., and Rasmussen, K., 2010. "Nonlinear buckling optimization of composite structures considering "worst" shape imperfections". International Journal of Solids and Structures, 47(22-23), NOV, pp. 31863202.

[5] Huang, H., and Han, Q., 2010. "Research on nonlinear postbuckling of functionally graded cylindrical shells under radial loads". Computers \& Structures, 92(6), MAY, pp. 1352-1357.

[6] Sampaio, R., and Soize, C., 2007. "Remarks on the efficiency of pod for model reduction in non-linear dynamics of continuous elastic systems". International Journal for Numerical Methods in Engineering, 72(1), pp. 22-45.

[7] Hollkamp, J. J., and Gordon, R. W., 2008. "Reduced-order models for nonlinear response prediction: Implicit condensation and expansion". Journal of Sound and Vibration, 318(4-5), DEC 23, pp. 1139-1153.

[8] Muryavov, A., and Rizzi, S., 2003. "Determination of nonlinear stiffness with application to random vibration of geometrically nonlinear structures". Computers \& Structures, 81, pp. 1513-1523. 
[9] Mignolet, M.-P., and Soize, C., 2008. "Stochastic reduced order models for uncertain geometrically nonlinear dynamical systems". Computer Methods in Applied Mechanics and Engineering, 197, pp. 3951-3963.

[10] Schenk, C., and Schuëller, G., 2003. "Buckling analysis of cylindrical shells with random geometric imperfections". International Journal of Nonlinear Mechanics, 38(7), pp. 1119-1132.

[11] Schenk, C., and Schuëller, G., 2007. "Buckling analysis of cylindrical shells with cutouts including random boundary and geometric imperfections". Computer Methods in Applied Mechanics and Engineering, 196(35-36), pp. 34243434.

[12] Soize, C., 2000. "A nonparametric model of random uncertainties for reduced matrix models in structural dynamics". Probabilistic Engineering Mechanics, 15(3), pp. 277-294.

[13] Soize, C., 2005. "A comprehensive overview of a nonparametric probabilistic approach of model uncertainties for predictive models in structural dynamics". Journal of Sound and Vibration, 288(3), pp. 623-652.

[14] Murthy, R., Wang, X., and Mignolet, M.-P., 2010. "Uncertainty-based experimental validation of nonlinear reduced order models". In Proceedings of the RASD 2010, Southampton, 12-14 July 2010.

[15] Capiez-Lernout, E., Soize, C., and Mignolet, M.-P., 2011. "Effect of structural uncertainties on the nonlinear elastic postbuckling behavior of a curved beam". In Proceedings of the Structural Dynamics EURODYN 2011, Leuven, Belgium, July 4-6, 2011.

[16] Capiez-Lernout, E., Soize, C., and Mignolet, M.-P., 2012. "Computational stochastic statics of an uncertain curved structure with geometrical nonlinearity in threedimensional elasticity". Computational Mechanics, 49(1), pp. 87-97.

[17] Soize, C., Capiez-Lernout, E., Durand, J.-F., Fernandez, C., and Gagliardini, L., 2008. "Probabilistic model identification of uncertainties in computational models for dynamical systems and experimental validation.”. Computer Mechanical Methods in Applied Mechanics and Engineering, 98(1), pp. 150-163.

[18] Capiez-Lernout, E., and Soize, C., 2008. "Robust updating of uncertain damping models in structural dynamics for low- and medium-frequency ranges.". Mechanical Systems and Signal Processing, 22(8), pp. 1774-1792.

[19] Sirovich, L., 1987. "turbulence and the dynamics of coherent structures .1. coherent structures". Quarterly of applied mathematics, 45(3), pp. 561-571.

[20] Lee, S.-H., 1992. MSC/Nastran Handbook for nonlinear analysis, version 67. Lee, S.-H. editor.

[21] Crisfield, M., 1997. Non-linear finite element analysis of solids and structures, Vol. 1 : essentials. John Wiley and Sons, Chichester.
[22] Yvonnet, J., Zahrouni, H., and Potier-Ferry, M., 2007. “A model reduction method for the post-buckling analysis of cellular microstructures". Computer Methods in Applied Mechanics and Engineering, 197, pp. 265-280.

[23] Anderson, E., Bai, Z., and Bischof, C., 1999. LAPACK User's Guide, Third Edition. SIAM, Philadelphia. 